\title{
One-Dimensional Nonequilibrium Radiation-Transport Equation under Diffusion Approximation and Its Discrete Scheme
}

\author{
Qi Gao, ${ }^{1}$ Chuanfei Zhang, ${ }^{2}$ Lin Zhou, ${ }^{2}$ Zhenghong Li, ${ }^{2}$ Zeqing Wu, ${ }^{3}$ Yu Lei, ${ }^{1}$ \\ Chunlai Zhang, ${ }^{1}$ and Xiaotao $\mathrm{Zu}^{1}$ \\ ${ }^{1}$ University of Electronic Science and Technology of China, Chengdu 610054, China \\ ${ }^{2}$ Institute of Nuclear Physics and Chemistry, CAEP, Mianyang 621900, China \\ ${ }^{3}$ Institute of Applied Physics and Computational Mathematics, Beijing 100088, China
}

Correspondence should be addressed to Qi Gao; fbc1980@163.com and Xiaotao Zu; xtzu@uestc.edu.cn

Received 20 March 2014; Accepted 1 June 2014; Published 24 June 2014

Academic Editor: Haiyan Xiao

Copyright (C) 2014 Qi Gao et al. This is an open access article distributed under the Creative Commons Attribution License, which permits unrestricted use, distribution, and reproduction in any medium, provided the original work is properly cited.

Based on the nonlocal thermodynamic equilibrium state and large optical thickness of plasma, we establish one-dimensional nonequilibrium radiation-transport equation from diffusion approximation. Through finite volume method, the discrete scheme of radiation-transport equation and the conditions for its definite solution are proposed. The reliability of radiation-transport equation and its discrete scheme is validated.

\section{Introduction}

Radiation-transport equation is one of the key equations which describe radiation and transport processes in plasma [1-4]. It is also a hot topic in numerical calculation of high energy density materials. Solving the radiation-transport equation is easy for optically thin material but difficult for optically thick material. In the latter case, most of studies assume plasma in local thermodynamic equilibrium state (LTE) and under nonequilibrium radiation field. Then the multigroup radiation-transport theory or diffusion approximation is employed using different radiation parameters in plasma [5-8]. This method has acquired a great success in ICF plasma and other fields; however, few theoretical studies on the radiation and transport processes in NLTE plasma have been reported so far $[9,10]$. Generally, the LTE assumption cannot always be met in plasma produced by experiments or under other conditions, and it will be more complex to solve the radiation-transport equation using nonlocal thermodynamic equilibrium plasma theory. This is because the induced radiation term appears in the equation. To solve this problem, we must simplify the dependency relationship between radiation and frequency or angle. The simplest and the most commonly used method is diffusion approximation, which is the lowest order approximation in spherical harmonics method. This method is an accurate description of the equation when plasma is optically thick and the radiation intensity is nearly isotropic [9].

Based on the character of large optical thickness of plasma under nonlocal thermodynamic equilibrium state, we establish one-dimensional nonequilibrium radiationtransport equation from diffusion approximation in this work. Besides, the discrete scheme of radiation-transport equation and the conditions for its definite solutions are proposed through finite volume method. The reliability of radiation-transport equation and its discrete scheme is also validated.

\section{Diffusion Approximation of Radiation- Transport Equation}

2.1. Radiation-Transport Equation under Diffusion Approximation. Generally, the radiation-transport equation can be described as $[11,12]$

$$
\frac{1}{c} \frac{\partial I_{v}}{\partial t}+\Omega \cdot \nabla I_{v}=j_{v}\left(1+\frac{c^{2}}{2 h v^{3}} I_{v}\right)-u_{v} I_{v} .
$$


In formula (1), $I_{v}$ is radiation intensity, $j_{v}=j_{b b}+j_{f f}+j_{f b}$ is the spontaneous emission coefficient, and $u_{v}=u_{b b}+u_{b f}+u_{f f}$ is absorption coefficient in plasma.

The dependency relationship between radiation and frequency or angle can be described as the preceding two items of spherical harmonics when the radiation intensity of plasma is nearly isotropic:

$$
I_{v}=\frac{1}{4 \pi} I_{0}(v)+\frac{3}{4 \pi} \Omega * I_{1}(v),
$$

where $I_{0}(v)$ is the isotropic part and $I_{1}(v)$ is the anisotropic part.

Combing (2) with (1) and integrating over angle, we can obtain the equation containing the isotropic part $I_{0}(v)$ :

$$
\frac{1}{c} \frac{\partial I_{0}(v)}{\partial t}+\nabla I_{1}(v)=4 \pi j_{v}\left(1+\frac{c^{2}}{8 \pi h v^{3}} I_{0}(v)\right)-u_{v} I_{0}(v) .
$$

Combing (2) with (1), multiplying both sides by $\Omega$, and integrating over angle, the equation containing the anisotropic part $I_{1}(v)$ can also be obtained:

$$
\frac{1}{c} \frac{\partial I_{1}(v)}{\partial t}+\frac{1}{3} \nabla I_{0}(v)=-\frac{1}{L_{v}} I_{1}(v) .
$$

The combination of (3) and (4) is the $P_{1}$ approximation. Basically, it is not easy to solve these two equations. Considering that radiation intensity is nearly isotropic and $I_{1}(v) * \Omega$ is much smaller than $I_{0}(v), I_{1}(v) * \Omega$ can be neglected and the problem will be further simplified.

Taking (4) into (3), we can obtain equation about $I_{0}(v)$ finally:

$$
\begin{aligned}
& \frac{1}{c} \frac{\partial I_{0}(v)}{\partial t}-\frac{1}{3} \nabla * L_{v} \nabla I_{0}(v) \\
& \quad=4 \pi j_{v}\left(1+\frac{c^{2}}{8 \pi h v^{3}} I_{0}(v)\right)-u_{v} I_{0}(v) .
\end{aligned}
$$

Replacing $I_{0}(v)$ with $\varepsilon_{v}\left(\varepsilon_{v}=(1 / c) \int_{4 \pi} I_{v} d \Omega\right)$, the equation can be described as

$$
\frac{\partial \varepsilon_{v}}{\partial t}-\nabla *\left(D_{v} \nabla \varepsilon_{v}\right)=4 \pi j_{v}\left(1+\frac{c^{3}}{8 \pi h v^{3}} \varepsilon_{v}\right)-c u_{v} \varepsilon_{v}
$$

Here, $D_{v}$ is the spectral diffusion coefficient. The radiationtransport equation considering the electron scattering progress is similar to (6) and only $D_{v}$ is different.

Under one-dimensional circle model, the radiationtransport equation can be described as

$$
\frac{\partial E_{v}}{\partial t}=\frac{\partial}{\partial r}\left(D_{v} \frac{\partial E_{v}}{\partial r}\right)+\left[\frac{4 \pi \eta_{0}}{v^{3}} j_{v}-c u_{v}\right] \cdot E_{v}+4 \pi j_{v} .
$$

2.2. The Conditions for Definite Solution to RadiationTransport Equation. To solve the established radiationtransport equation, the initial and boundary conditions are necessary. The initial condition is simple, which is the radiation spectrum of plasma at centre and $t=0$.
The boundary conditions are very complex. The boundary conditions can be described as

$$
E_{v}=\Gamma_{v}, \quad n \cdot \Omega<0,
$$

where $n$ is the outer normal vector of unit on system surface.

Under diffusion approximation, the restrict boundary conditions are not satisfied. A weighting function is defined to solve this problem. The boundary conditions can be described as [11, 13]

$$
\begin{aligned}
& \int_{n \cdot \Omega<0}[ \frac{1}{4 \pi} I_{0}\left(r_{s}, v, \Omega, t\right)+\frac{3}{4 \pi} \Omega * I_{1}\left(r_{s}, v, \Omega, t\right) \\
&\left.-\Gamma\left(r_{s}, v, \Omega, t\right)\right] w(\Omega) d \Omega .
\end{aligned}
$$

Taking into account Marshak vacuum boundary condition, that is, $w(\Omega)=n \cdot \Omega$, there is no photon coming into system through surface. Thus, the following formula can be obtained:

$$
\left.J^{-}\right|_{r=R}=\left[-\frac{c}{4} E_{v}-\frac{D_{v}}{2} \frac{\partial E_{v}}{\partial x}\right]_{r=R}=0 .
$$

The radiation intensity is nearly isotropic at the centre of the system, and we obtain

$$
\left[\frac{D_{v}}{2} \frac{\partial E_{v}}{\partial x}\right]_{r=R}=0
$$

\section{Conservation Finite Difference Scheme of $1 D$ Unstable Radiation-Transport Equation}

The radiation-transport equation belongs to variable coefficient parabolic equations with the third boundary conditions in mathematics. We can acquire its conservation finite difference scheme through finite volume method [14]. According to finite difference theory, the time and space can be divided into meshes, where $t_{n}=n k(n=1,2, \ldots,[T / k])$ and $r_{j}=$ $j h(j=1,2, \ldots,[R / h])$. Using $E_{v j}^{n}$ to represent the exact value of $E_{v}$ at mesh point $\left(x_{j}, t_{n}\right)$, defining $D_{v}(r, t)\left(\partial E_{v} / \partial r\right)=$ $Z_{v}(r, t)$ and taking it into (7), we can get

$$
\frac{\partial Z_{v}}{\partial r}=\frac{\partial E_{v}}{\partial t}+\left[\frac{4 \pi \eta_{0}}{v^{3}} j_{v}-c u_{v}\right] E_{v}+4 \pi j_{v}=k\left(E_{v}, x, t\right) .
$$

Integrating the above equation over $\left(r_{j}-(h / 2), r_{j}+(h / 2)\right)$ region, we can obtain

$$
Z\left(r_{j}+\frac{h}{2}, t\right)-Z\left(r_{j}-\frac{h}{2}, t\right)=\int_{r_{j}-h / 2}^{r_{j}+h / 2} k\left(E_{v} ; \lambda, t\right) d \lambda .
$$

Integrating (13) over $\left(r_{j-1 / 2}, r\right)$ region, multiplying it by $1 / D_{v}(r, t)$, and integrating it over $\left(r_{j-1}, r_{j}\right)$ region, the following equation can be obtained:

$$
\begin{aligned}
Z_{v}\left(r_{j-1 / 2}, t\right)= & \frac{E_{v}\left(r_{j, t}\right)-E_{v}\left(r_{j-1, t}\right)}{\int_{r_{j-1}}^{r_{j}}\left(d r / D_{v}(r, t)\right)}-\frac{1}{\int_{r_{j-1}}^{r_{j}}\left(d r / D_{v}(x, t)\right)} \\
& \times \int_{r_{j-1}}^{r_{j}} \frac{d r}{D_{v}(r, t)} \int_{r_{j-1 / 2}}^{r} k\left(E_{v} ; \lambda, t\right) d \lambda .
\end{aligned}
$$


Similarly, we can get

$$
\begin{aligned}
Z_{v}\left(r_{j+1 / 2}, t\right)= & \frac{E_{v}\left(r_{j+1, t}\right)-E_{v}\left(r_{j, t}\right)}{\int_{r_{j}}^{r_{j+1}}\left(d r / D_{v}(x, t)\right)}-\frac{1}{\int_{r_{j}}^{r_{j+1}}\left(d r / D_{v}(r, t)\right)} \\
& \times \int_{r_{j}}^{r_{j+1}} \frac{d r}{D_{v}(r, t)} \int_{r_{j+1 / 2}}^{r} k\left(E_{v} ; s, t\right) d s .
\end{aligned}
$$

Combining (14) and (15) with (12), integrating it over $\left(t_{n}, t_{n+1}\right)$ region, introducing a parameter $\theta$, and using some approximate integral formula in (9), we can obtain the discrete scheme of (7) that is compatible with the one-dimensional radiation-transport equation:

$$
\begin{aligned}
& \frac{E_{v j}^{n+1}-E_{v j}^{n}}{k} \\
& =\frac{(1-\theta)}{h^{2}}\left[D_{v j-(1 / 2)}^{n+1} E_{\gamma_{j-1}}^{n+1}\right. \\
& \quad-\left(D_{v j-(1 / 2)}^{n+1}+D_{v j+(1 / 2)}^{n+1}\right) E_{v j}^{n+1} \\
& \left.+D_{v j+(1 / 2)}^{n+1} E_{v j+1}^{n+1}\right] \\
& +\frac{\theta}{h^{2}}\left[D_{v j-(1 / 2)}^{n} E_{\gamma_{j-1}}^{n}\right. \\
& \left.\quad-\left(D_{v j-(1 / 2)}^{n}+D_{v j+(1 / 2)}^{n}\right) E_{v j}^{n}+D_{v j+(1 / 2)}^{n} E_{v j+1}^{n}\right] \\
& +(1-\theta)\left(\frac{4 \pi \eta_{0}}{v^{3}} j_{v j}^{n+1}-c u_{v j}^{n+1}\right) E_{v j}^{n+1} \\
& +\theta\left(\frac{4 \pi \eta_{0}}{v^{3}} j_{v j}^{n}-c u_{v j}^{n}\right) E_{v j}^{n} \\
& +4 \pi(1-\theta) j_{v j}^{n+1}+4 \pi j_{v j}^{n} .
\end{aligned}
$$

The truncation error for (16) is

$$
k^{-1} T_{j}^{n}=-k\left(\frac{1}{2}-\theta\right)\left(\frac{\partial^{2} E_{v}}{\partial t^{2}}\right)+O\left(k^{2}+h^{2}\right) .
$$

When the value of $\theta$ is 1,0 , and $1 / 2$, the corresponding finite difference scheme for formula (17) is explicit, implicit with an accuracy of $O\left(k+h^{2}\right)$, and Crank-Nicholson scheme with an accuracy of $O\left(k+h^{2}\right)$, respectively.

Applying Taylor expansion to the boundary conditions, the discrete scheme for the boundary conditions can be provided. At point $r=R$ and $r=0$, the following equations can be obtained:

$$
\begin{gathered}
\left(D_{v J-(1 / 2)}^{n+1}+D_{v J-(3 / 2)}^{n+1}\right) E_{J-1}^{n+1}=\left(2 D_{v J}^{n+1}+h c\right) E_{\gamma_{J}}^{n+1}, \\
\left(D_{v 1 / 2}^{n+1}+D_{v 3 / 2}^{n+1}\right) E_{v 1}^{n+1}-2 D_{v 0}^{n+1} E_{v 0}^{n+1}=0 .
\end{gathered}
$$

Combining (17) and its boundary condition equations (18), the radiation-transport equation can be solved by the chase after method.

\section{A Test Example}

In this section, a radiation spectrum which is calculated by NLTE model based on digital level model [15] is used to test the reliability of radiation-transport equation under diffusion approximation. In this example, variation of spectrum shape and intensity with transition time and thickness of plasma is analyzed. The electronic temperature $T_{e}$ is taken as $450 \mathrm{eV}$, and the electron density $N_{e}$ is taken as $1 \times 10^{20} \mathrm{~cm}^{-3}$. The effects of external radiation source are not taken into account.

Essentially, the radiation-transport process of photons is the absorption of photons in plasma and superposition with other photons radiated by plasma. The calculated results for photon transport in plasma with a radius of $250 \mathrm{um}$ are shown in Figures 1 and 2. Figure 1 is the subset of variation of spectrum shape with time for plasma radiation at boundary points. Figure 2 shows the change of partial transition intensity ratio as a function of time. Ly $\alpha \mathrm{IC}$ are mainly composed of three spectrum lines coming from $2 p^{2} \rightarrow 1 s^{1} 2 p^{1}$ radioactive transition; He $\alpha$ IC are mainly comprised of spectrum lines coming from $1 s^{1} 2 p^{2} \rightarrow 1 s^{2} 2 p^{1}$ radioactive transitions; and $\mathrm{He} \alpha$ + IC are mainly constituted by spectrum lines from $1 s^{1} 2 p^{2} \rightarrow 1 s^{2} 2 p^{1}$ and $1 s^{1} 2 p^{1} 3 p^{1} \rightarrow$ $1 s^{2} 3 p^{1}$ radioactive transitions.

As shown in Figures 1 and 2, the radiation spectrum lines vary with time gradually and finally reach equilibrium states. For plasma with a radius of $250 \mathrm{um}$, the intensity of radiation spectrum increases gradually if transition time is smaller than $5 \times 10^{-3}$ ns. Different transition lines increase variously, and the shape of radiation spectrum has an obvious change. With the increase in transition time, the $\mathrm{K}$ shell resonance radiation intensity achieves dynamic equilibrium and does not increase anymore as the transition time is larger than $5 \times 10^{-3} \mathrm{~ns}$. The intensity of satellite lines increases continuously until the transition time is larger than $1.0 \times 10^{-2} \mathrm{~ns}$.

These results can be explained physically as follows. The radiation at certain boundary points is only contributed by self-radiation of plasma initially. Subsequently, the nearby radiation arrives at the boundary points after absorption by plasma. In this case, the radiation spectrum at boundary points is the superposition of two parts. As the transition time increases, more and more radiations arrive at the boundary points after absorption by plasma and the total radiation effects at boundary points are the superposition of these radiations. Due to the different absorption coefficient of transition lines, the absorption extent of transition lines through the same thickness of plasma is different, and the shape of radiation spectrum at boundary points changes obviously during transition process. With increasing transition time, some transition lines with superior absorption coefficient are absorbed completely before they reach boundary points, while the radiations with minor absorption coefficient can still arrive at boundary points. If transition time is so large that all radiations arrive at the boundary points after absorption or are absorbed completely, the radiation spectrum will achieve dynamic equilibrium states. Generally speaking, in the process reaches a dynamic equilibrium state, the changement of relative strength for 

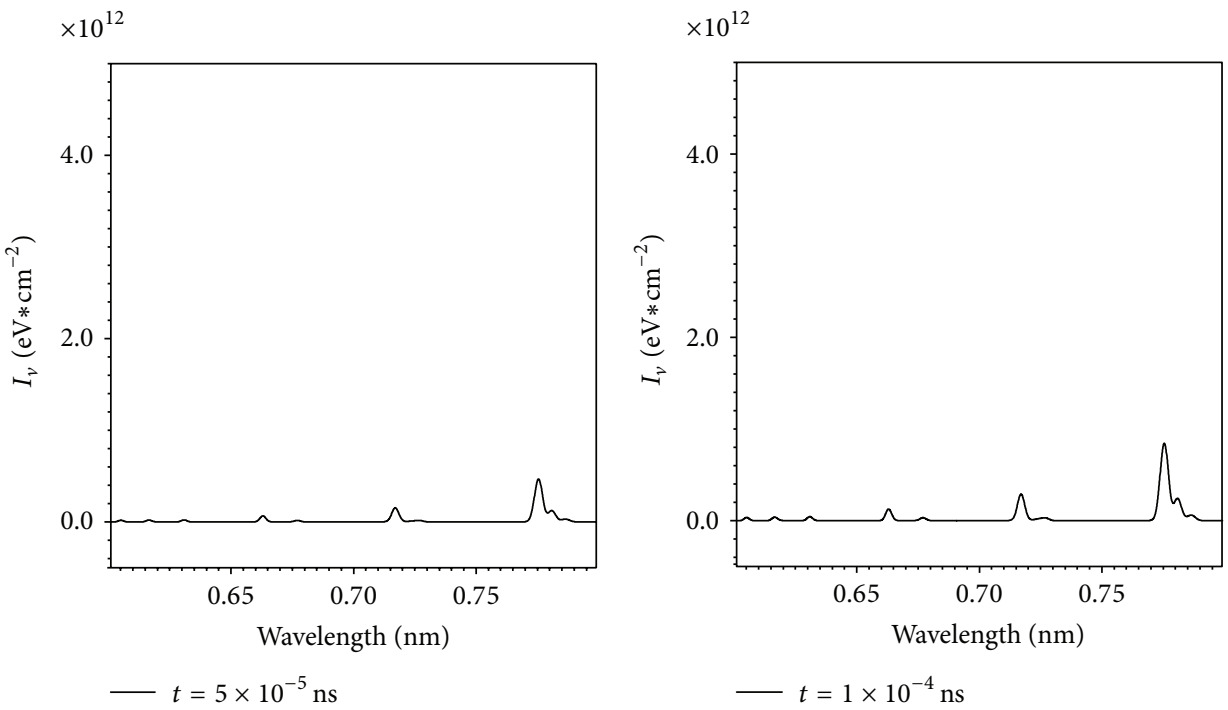

(a)

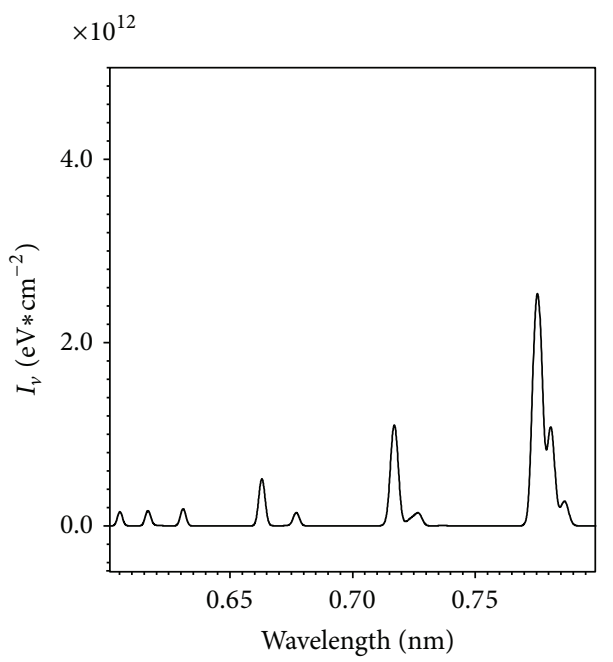

(b)

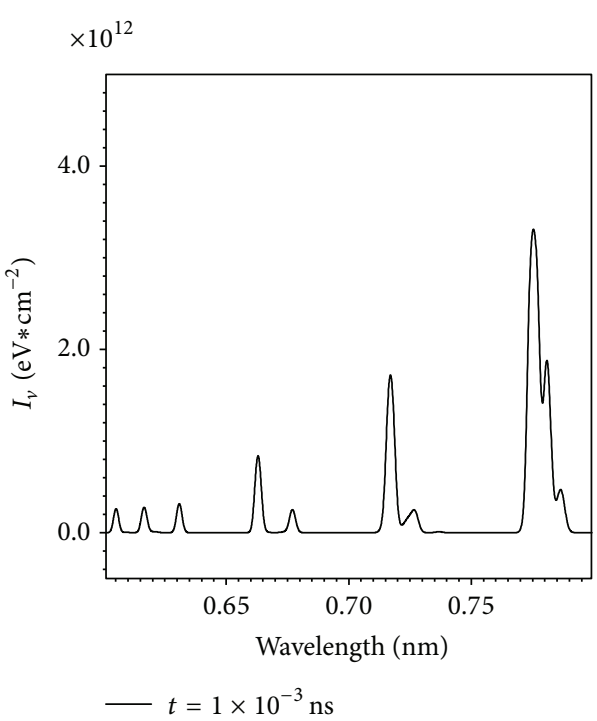

(d)

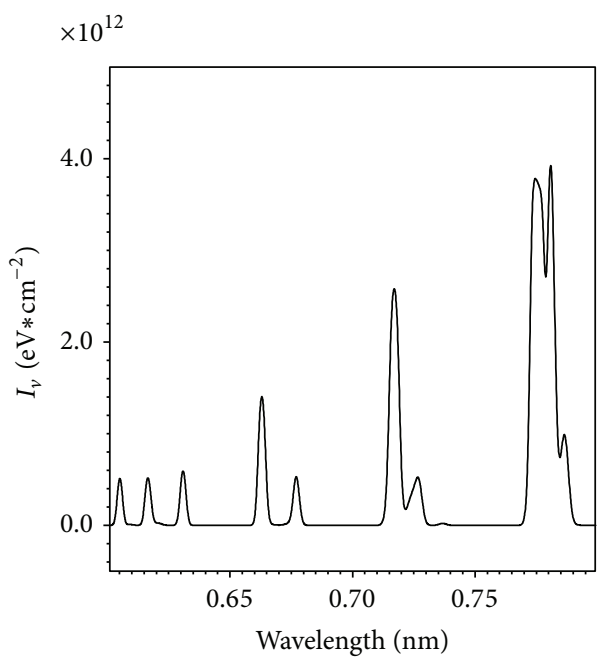

$-t=5 \times 10^{-3} \mathrm{~ns}$

(e)

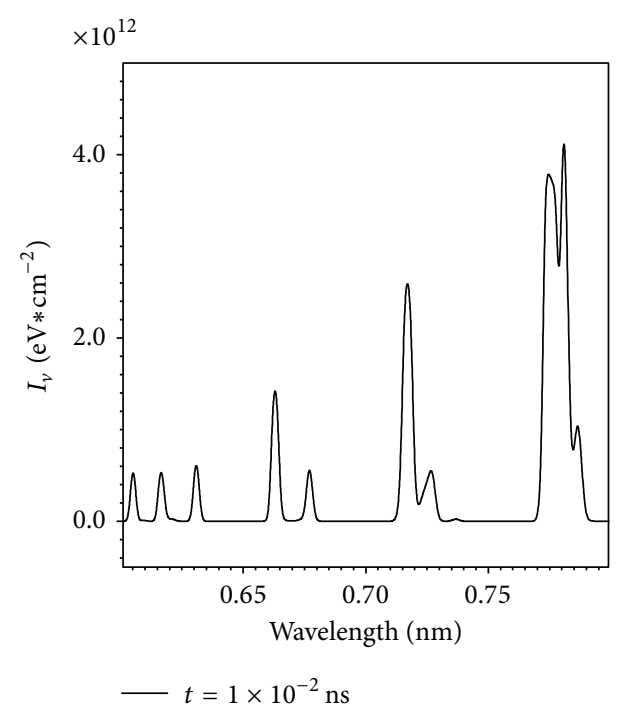

(f)

FIGURE 1: Subset of variation of radiation spectrum. 


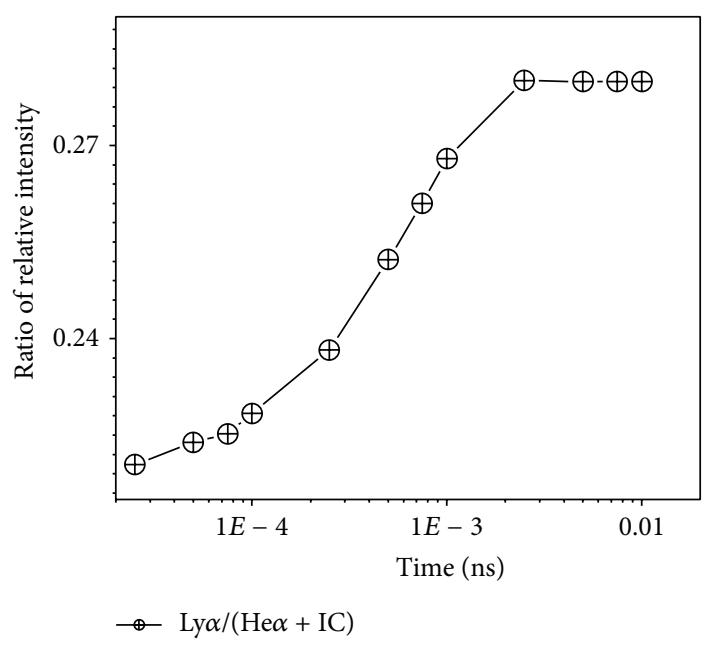

(a)

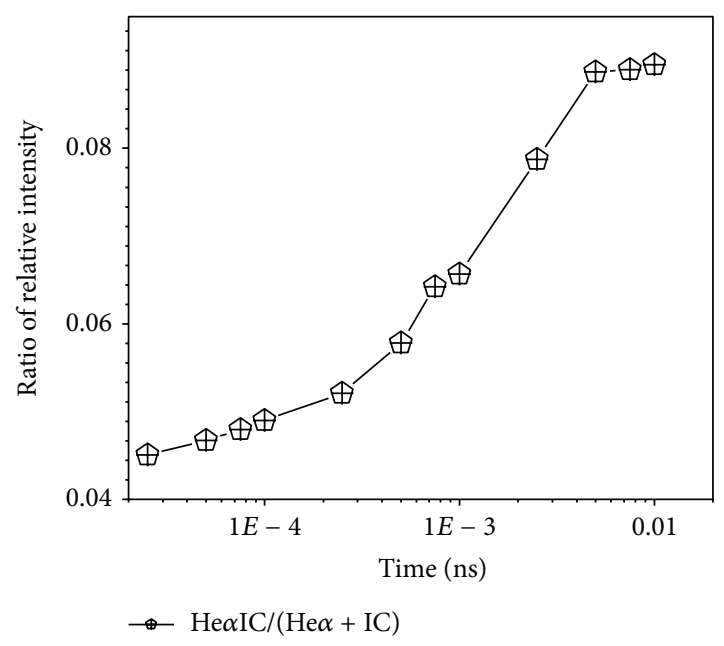

(c)

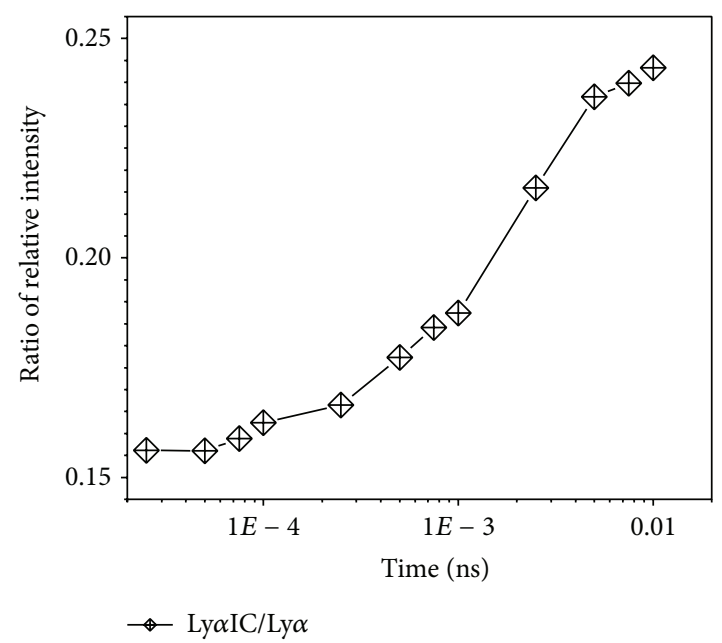

(b)

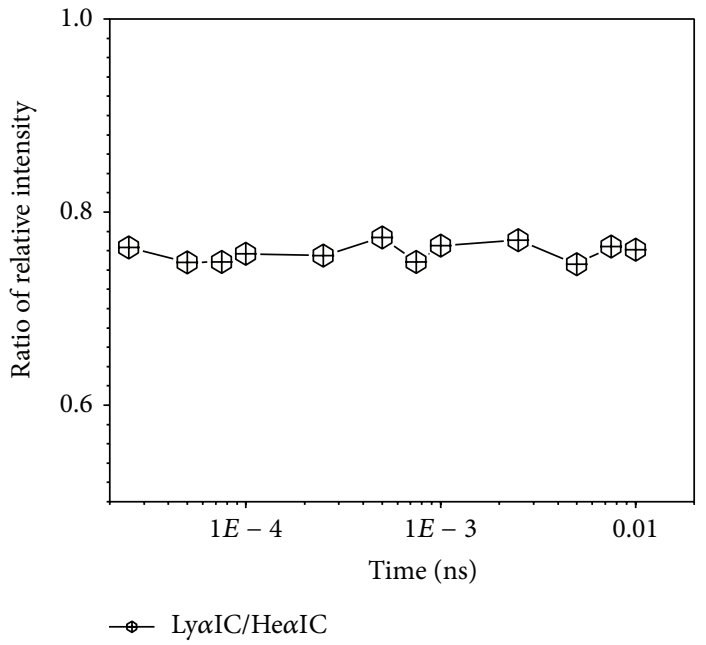

(d)

FIGURE 2: Variation of some transition intensity ratio with time.

$\mathrm{K}$ shell resonan ceradiation in $\mathrm{Al}$ plasma is much larger than their satellite lines because of their superior absorption coefficient.

The transport time needed to achieve dynamic equilibrium states is different for plasma with different radii. The final radiation spectrum at boundary points is also highly related to the radius of the plasma. Figures 3 and 4 present the radiation spectrum at boundary points and some radiation intensity ratio for plasma with different radii. In the calculations the transport time is large enough to ensure all radiation reaching dynamic equilibrium states. Combining Figures $1-4$, it is found that the $\mathrm{K}$ shell resonance radiation of Al plasma is sensitive to the absorption effects as $T_{e}$ is $450 \mathrm{eV}$ and $N_{e}$ is $1 \times 10^{20} \mathrm{~cm}^{-3}$. When the transport time and the radius of plasma change, the radiation intensity ratios change obviously, while the satellite lines are not so sensitive to the absorption effects. The radiation intensity ratios nearly remain unchanged when the transport time and plasma radius vary.
These results show that the radiation-transport equation proposed in this study can be explained physically and is consistent with the basic law of photon transport in plasma with large optical thickness.

\section{Conclusion}

Based on the nonlocal thermodynamic equilibrium state and large optical thickness of plasma, a simplification and discrete method to radiation-transport equation is proposed in this work. One-dimensional nonequilibrium radiation-transport equation is deduced from diffusion approximation and the conditions for its definite solution are proposed. The discrete scheme of radiation-transport equation and the conditions for its definite solution are obtained through finite volume method. The reliability of radiation-transport equation and its discrete scheme has been tested by NLTE model based on digital level. The calculations show that the deductions 

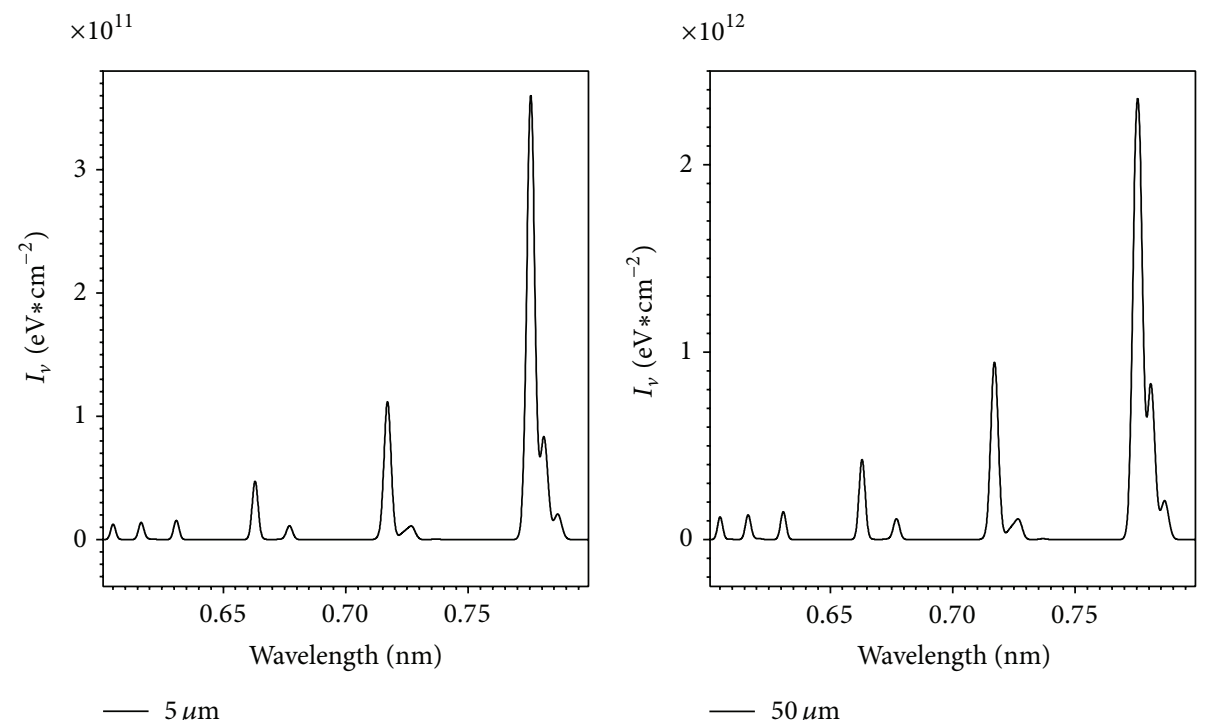

(a)

(b)
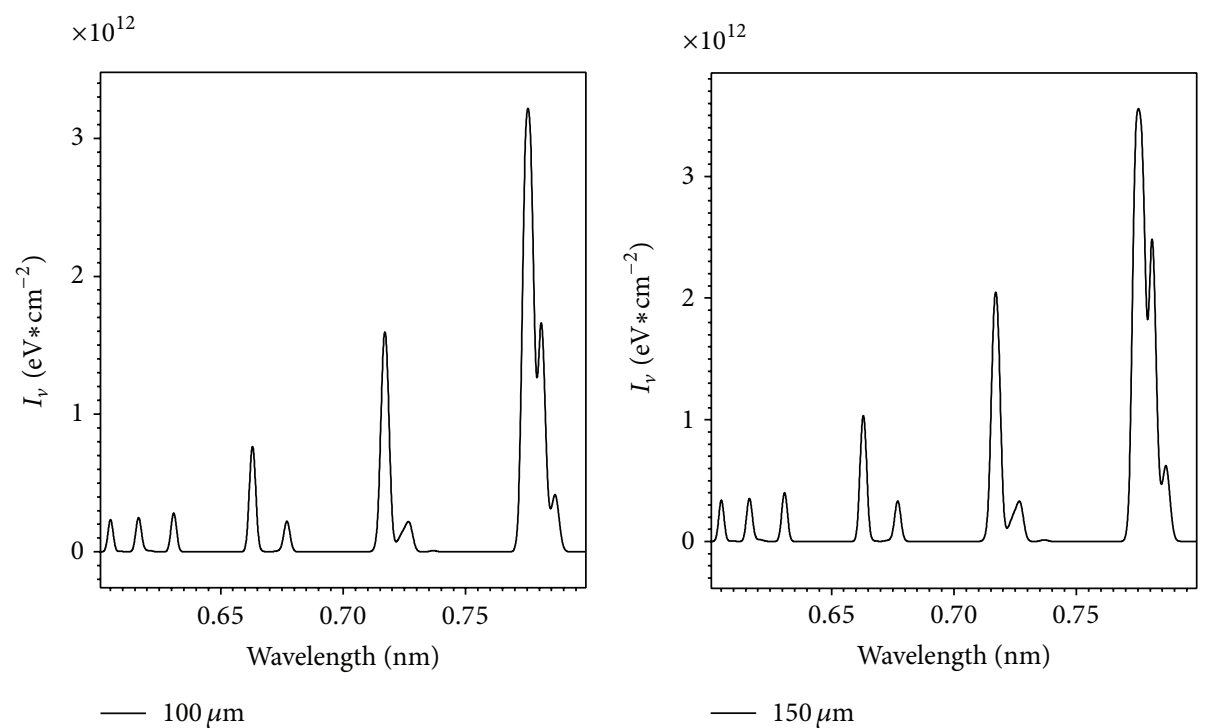

(c)

(d)

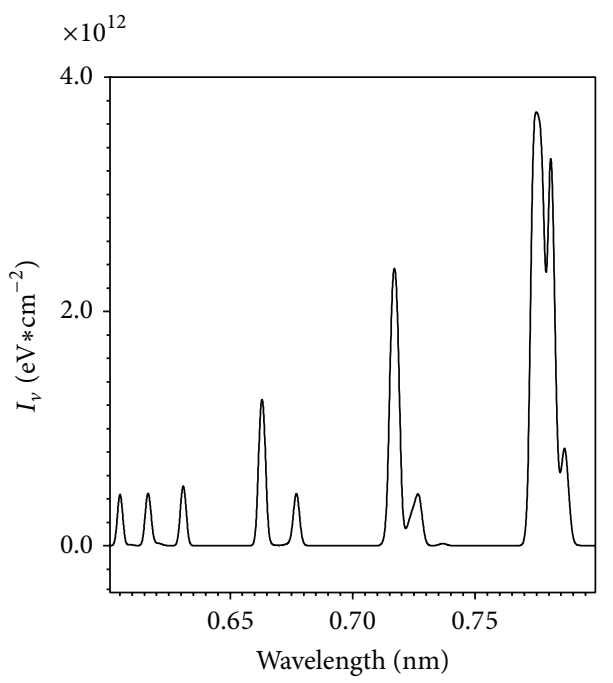

$\times 10^{12}$

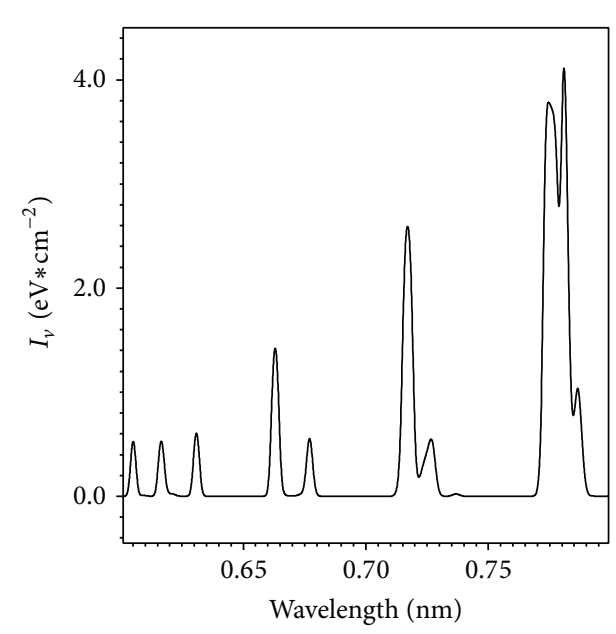

$-200 \mu \mathrm{m}$

$-250 \mu \mathrm{m}$

(e)

(f)

FIGURE 3: Radiation spectrum of different plasma at boundary points. 


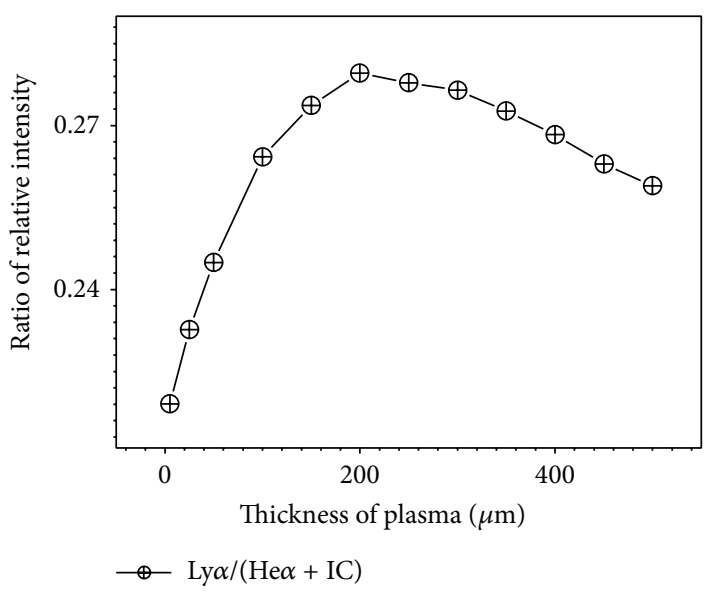

(a)

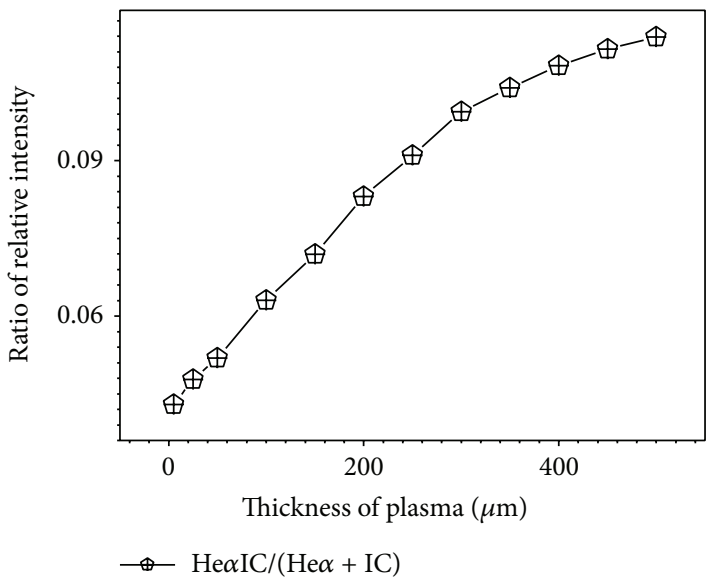

(c)

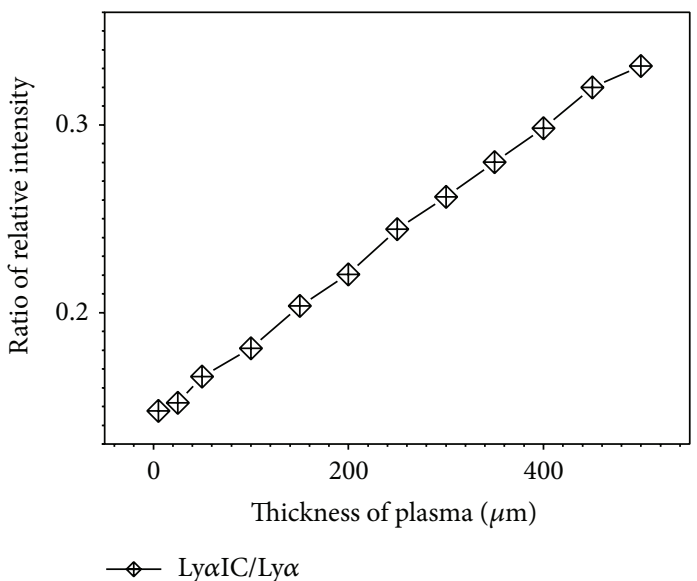

(b)

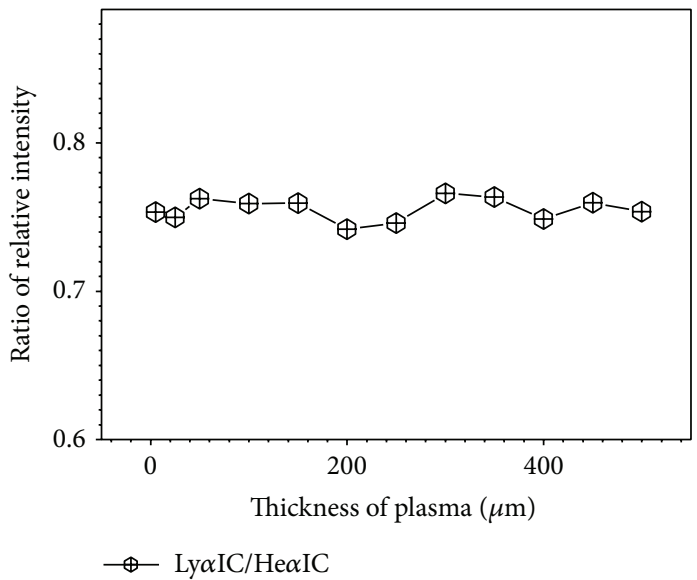

(d)

FIGURE 4: Radiation intensity ratio of different plasma at boundary points.

from the diffusion approximation and the discrete method are reliable. Also, the calculated results are consistent with the basic law of photon transport in optically thick plasma.

It should be pointed out that only the reliability of radiation-transport equation under diffusion approximation can be validated. When plasma is under NLTE state, besides solving the equation itself, radiation parameters should also be calculated, which is difficult in some cases. To validate the reliability of calculations of radiation-transport process in plasma, both the radiation-transport equation and related population equation should be considered. The conclusion will be more convincing only when the calculated results are verified by experiments.

\section{Conflict of Interests}

The authors declare that there is no conflict of interests regarding the publication of this paper.

\section{Acknowledgments}

This work was supported financially by the NSAF Joint Foundation of China (no. U1230124) and the Scientific Research
Foundation for the Central Universities of China (Grant no. 2014NZYQN14).

\section{References}

[1] D. Mihalas and B. W. Mihalas, Foundations of Radiation Hydrodynamics, Oxford University Press, Oxford, UK, 1984.

[2] J. S. Truelove, "Discrete-ordinate solutions of the radiation transport equation," Journal of Heat Transfer, vol. 109, no. 4, Article ID 5476424, 1987.

[3] Y. Maron, A. Starobinets, V. I. Fisher et al., "Pressure and energy balance of stagnating plasmas in z-pinch experiments: implications to current flow at stagnation," Physical Review Letters, vol. 111, no. 3, Article ID 035001, 2013.

[4] S. G. Garanin, A. V. Ivanovsky, and L. S. Mkhitariyan, "An ICF system based on $\mathrm{Z}$-pinch radiation produced by an explosive magnetic generator," Nuclear Fusion, vol. 51, no. 10, Article ID 103010, 2011.

[5] Y. W. Li, T. G. Feng, and D. X. Lai, "A variable multigroup method for calculation in non equilibrium radioactive transfer," Chinese Journal of Computation Physics, vol. 11, no. 3, pp. 328336, 1994. 
[6] T. M. Song and J. M. Yang, "One-dimensional simulation of radiation transport in three dimensional cylinder," Acta Physica Sinica, vol. 62, no. 1, Article ID 015210, 2013.

[7] X. Hang, J. Li, and G. Yuan, "Convergence analysis on splitting iterative solution of multi-group radiation diffusion equations," Chinese Journal of Computational Physics, vol. 30, no. 1, pp. 111119, 2013.

[8] J. W. Li and J. H. Li, "The multi-group approximation for radiation diffusion equations," in Proceedings of the 5th National Youth Computational Physics Symposium, Qingdao, China, July 2008.

[9] B. Duan, Z. Q. Wu, J. Yan et al., "Diagnosis for ginertial confinement fusion plasma," Journal of Atomic and Molecular Sciences, vol. 24, no. 1, 2007.

[10] B. Duan, Z. Q. Wu, and J. G. Wang, "X spectrum diagnosis for ginertial confinement fusion plsma," Science in China G: Physics, Mechanics \& Astronomy, vol. 34, no. 1, pp. 41-54, 2009.

[11] H. M. Peng, Radiation Transport and Radiation Hydrodynamics in Plasmas, National Defense Industrial Press, Beijing, China, 1991.

[12] S. C. Li, High Temperature Radiation Physics and the Quantum theory of Radiation, National Defence Industry Press, Beijing, China, 1980.

[13] Z. Q. Huang and E. J. Ding, Transport Theory, Science Press, Beijing, China, 2008.

[14] S. C. Zhang, Finite Difference Method for Fixed Solution Problem of Parabolic Equations, Science Press, Beijing, China, 2010.

[15] Q. Gao, Z. Q. Wu, C. F. Zhang et al., "The NLTE simulation of Z-pinch Al plasma radiation," Acta Physica Sinica, vol. 61, no. 1, Article ID 015201, 2012. 

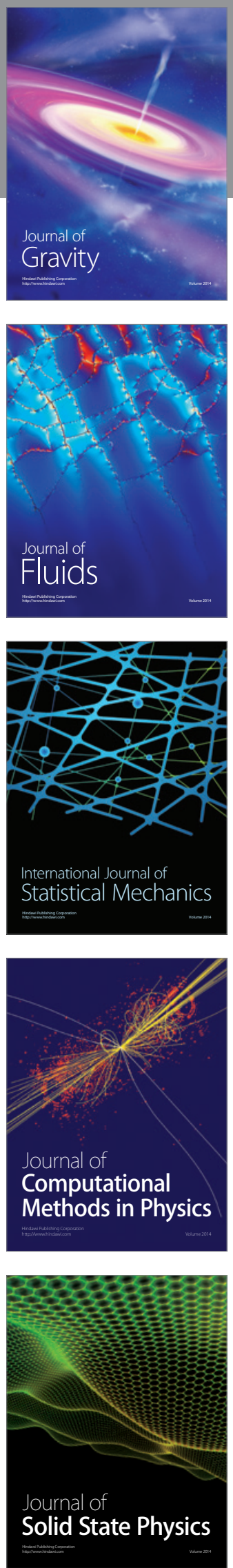

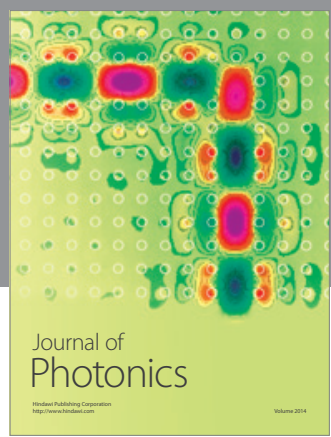

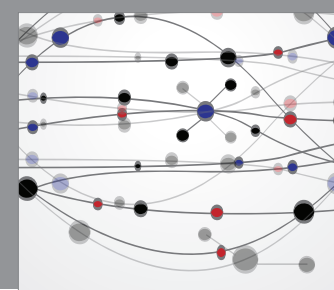

The Scientific World Journal

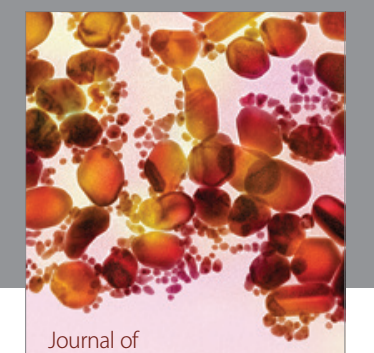

Soft Matter
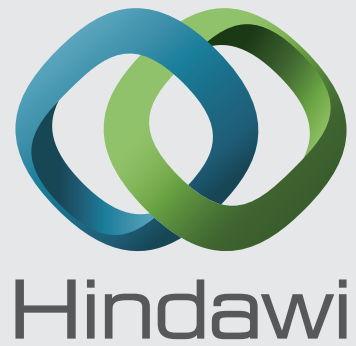

Submit your manuscripts at

http://www.hindawi.com
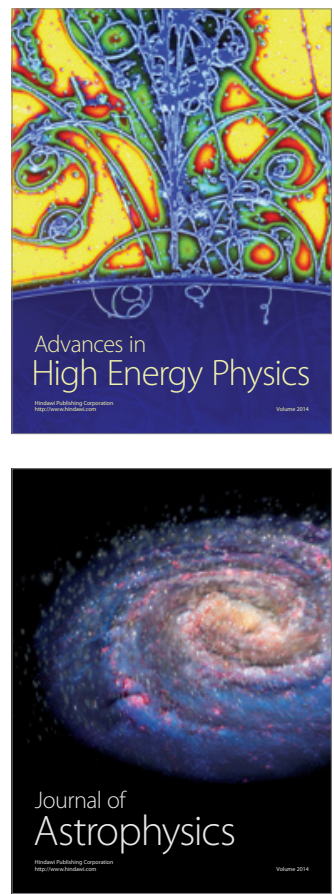
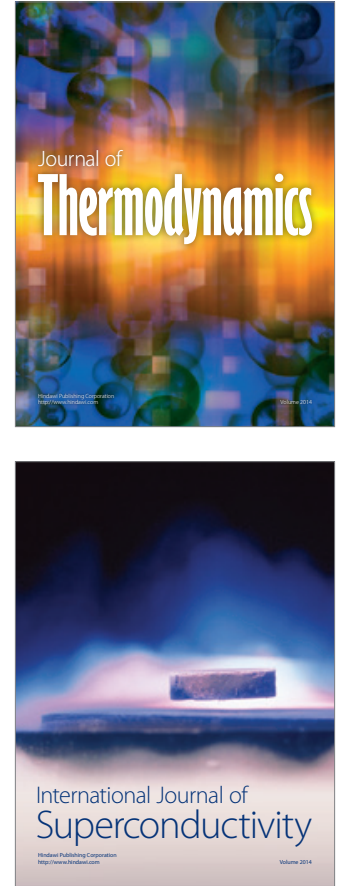
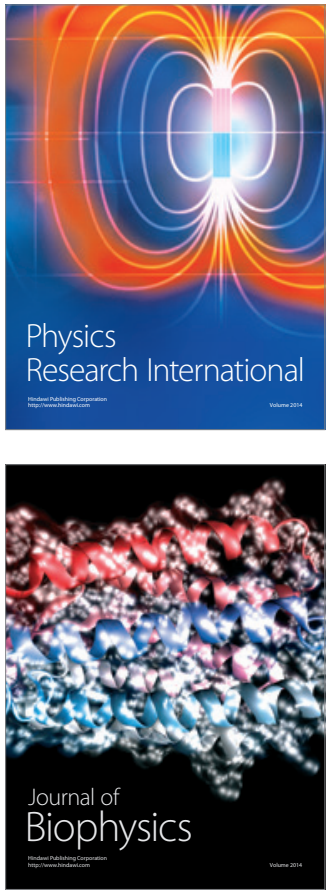
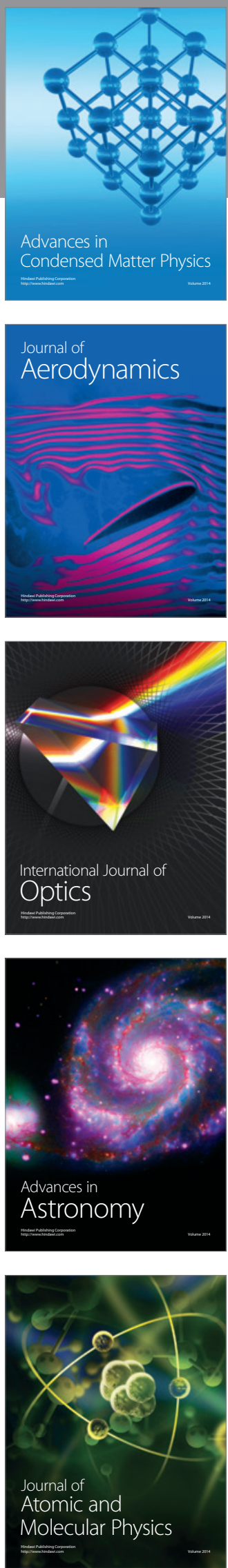